\title{
Hiperbilirrubinemia severa en Recién Nacidos, factores de riesgo y secuelas neurológicas
}

\author{
Severe hyperbilirubinemia in newborns, risk factors and neurological outcomes
}

\author{
Stephanie Campbell Wagemann ${ }^{\mathrm{a}}$, Patricia Mena Nannig ${ }^{\mathrm{b}}$
}

aResidente de Neonatología, División de Pediatría, Pontificia Universidad Católica de Chile

bUnidad de Neonatología Centro Asistencial Dr. Sotero del Rio, y División de Pediatría, Pontificia Universidad Católica de Chile, Santiago, Chile.

Recibido: 11 de junio de 2018; Aprobado: 7 de diciembre de 2018

\begin{abstract}
Resumen
Introducción: La hiperbilirrubinemia es altamente prevalente en los recién nacidos, con riesgo de compromiso neurológico con bilirrubinemias mayor a 20-25 mg/dl. Esta progresión es prevenible con detección y tratamiento precoz. Objetivo: Describir incidencia y factores asociados en pacientes hospitalizados con hiperbilirrubinemia mayor de $20 \mathrm{mg} / \mathrm{dl}$, y el seguimiento de casos sintomáticos durante hospitalización. Pacientes y Método: Estudio retrospectivo de pacientes con hiperbilirrubinemia severa, entre el 2013 y 2016. Se evaluaron factores de riesgo, estratificándose por nivel de bilirrubina, edad de ingreso y edad gestacional. Se compararon los datos con test exacto de Fisher, chi cuadrado y riesgo relativo (RR) en una base de excel, con un error alfa de un $\mathrm{p}<0.05$. Los datos fueron obtenidos a través de la epicrisis electrónica y de la ficha de control a nivel secundarios. Resultados: Durante el periodo, de 25.288 recién nacidos vivos (RNV), 593 se hospitalizaron por hiperbilirrubinemia mayor de $20 \mathrm{mg} / \mathrm{dl}, 1$ por cada $42 \mathrm{RNV}$; y 59 con bilirrubinemia mayor a $25 \mathrm{mg} / \mathrm{dl}, 1$ por cada 428 RNV. La hiperbilirrubinemia fue más frecuente en varones, con RR 1,22 (IC 95\% 1,04-1,44) y en pretérminos tardíos, con un RR 2,39 (IC 95\% 1,96-2,93) comparado con RN de término. En los ingresados con más de 4 días, el principal factor asociado fue la baja de peso excesiva, y en los primeros 3 días, la incompatibilidad de grupo clásico. Tres de 10 pacientes con encefalopatía aguda, persistieron con compromiso neurológico, lo que significa 11,8 por 100.000 nacidos vivos. Conclusiones: Los principales factores de riesgo para desarrollar hiperbilirrubinemia severa fueron prematurez, baja de peso excesiva, incompatibilidad de grupo clásico y sexo masculino. Estos hallazgos permiten focalizar la atención en grupos de riesgo y disminuir la probabilidad de daño neurológico.
\end{abstract}

Palabras clave: Hiperbilirrubinemia severa; prematurez; encefalopatía por bilirrubina; incompatibilidad grupo clásico 


\section{Abstract}

Introduction: Hyperbilirubinemia is highly prevalent in newborns, with risk of neurological involvement with bilirubinemia higher than 20 to $25 \mathrm{mg} / \mathrm{dl}$. This progression is preventable with early detection and treatment. Objective. To describe the incidence and associated factors in hospitalized patients with hyperbilirubinemia higher than $20 \mathrm{mg} / \mathrm{dl}$, and the follow-up of symptomatic cases during hospitalization. Patients and Method: Retrospective study of patients with severe hyperbilirubinemia, between 2013 and 2016. Risk factors were evaluated, stratifying by bilirubin level, admission age, and gestational age. The data were compared with Fisher's exact test, chi-square test, and relative risk $(\mathrm{RR})$ in an Excel database, with an alpha error of $\mathrm{p}<0.05$. The data were obtained from the electronic discharge summary and the medical record of secondary level follow-up. Results: During the studied period, out of 25,288 live newborns (NB), 593 were hospitalized due to hyperbilirubinemia higher than $20 \mathrm{mg} / \mathrm{dl}$, one per each 42 live NB; and 59 with bilirubinemia higher than $25 \mathrm{mg} / \mathrm{dl}$, one per each 428 live NB. Hyperbilirubinemia was more frequent in males, with RR 1.22 (95\% CI 1.04-1.44), and in late preterm newborns, with RR 2.39 (95\% CI 1.96-2.93) compared with term NB. In those admitted with more than four days, the main associated factor was excessive weight loss, whereas in the first three days was classic group incompatibility. Three of ten cases with acute encephalopathy persisted with neurological involvement, which means 11.8 per 100,000 live births. Conclusions: The main risk factors for developing severe hyperbilirubinemia were prematurity, excessive weight loss, classic group incompatibility, and male sex. These findings allow to focus attention on risk groups and decrease the probability of neurological damage.

\section{Keywords:}

Severe hyperbilirubinemia; prematurity; bilirubin encephalopathy; classic group incompatibility

\section{Introducción}

La ictericia es altamente prevalente en el recién nacido (RN), pudiendo afectar hasta $60-80 \%$ de estos ${ }^{1-3}$. Se considera hiperbilirrubinemia (HBR) cuando la bilirrubina sérica es mayor al percentil 95 para la edad y en general, se considera severa cuando los niveles superan los 20 o $25 \mathrm{mg} / \mathrm{dl}^{4,5}$.

La bilirrubina es un importante antioxidante, que posee un fino sistema regulador que mantiene los niveles estables, pero este puede verse afectado por diferentes causas, llevando a un aumento de la bilirrubina $^{6}$. A medida que aumentan los niveles de bilirrubina, existe el riesgo de desarrollar toxicidad neurológica o encefalopatía por bilirrubina. Este espectro abarca a la encefalopatía aguda y crónica por bilirrubina; y la disfunción neural aislada ${ }^{7}$. La encefalopatía aguda puede ir desde una succión débil hasta el compromiso neurológico severo con estupor profundo y opistótonos. La encefalopatía crónica o kernicterus es una entidad neurológica devastadora caracterizado por parálisis cerebral atetósica, con paresia oculomotora, displasia del esmalte dental y neuropatía auditi$\mathrm{va}^{8-11}$. El sistema auditivo es particularmente sensible a los efectos de la bilirrubina, pudiendo generar desde alteraciones en el procesamiento del habla hasta sordera profunda. El daño auditivo por bilirrubina requiere una ventana temporal, presentándose cuando las células están en pleno desarrollo, en la formación de circuitos neuronales, por lo que los prematuros tienen mayor riesgo. Además, las vías sensitivas se mielinizan antes que las motoras, esto lleva a que el kernicterus con daño auditivo predominante se ve más comúnmente en los menores de 34 semanas, en contraste con el tipo motor clásico que es más frecuente en los $\mathrm{RN}$ de término ${ }^{7,12,27}$. Esto puede ocurrir a niveles que antes no eran considerados como dañinos, pero en general, con niveles de bilirrubina mayores a $20 \mathrm{mg} / \mathrm{dl}$, es necesario el seguimiento auditivo a largo plazo ${ }^{6,7,12}$. Se ha descrito que más de la mitad de los RN con bilirrubina mayor a $30 \mathrm{mg} / \mathrm{dl}$ desarrollan secuelas neurológicas ${ }^{26}$.

La fototerapia y la exsanguineotransfusión han llevado a una reducción de la encefalopatía aguda y crónica por bilirrubina ${ }^{6,9}$. Con la prevención de la isoinmunosensibilización por Rh, el uso de inmunoglobulina y la efectividad de la fototerapia se ha disminuido marcadamente la necesidad de exsanguineotransfusión, que es un procedimiento invasivo, no libre de riesgos ${ }^{4,9}$.

A pesar de la existencia de tratamiento eficiente, el riesgo de HBR severa y sus consecuencias se observa con un amplio rango de incidencia a nivel mundial, dependiendo de las estrategias de control utilizadas ${ }^{4,9,13,14}$.

El objetivo de este trabajo es describir la incidencia y factores de riesgo asociados a bilirrubinemia igual o mayor a $20 \mathrm{mg} / \mathrm{dl}$, y la evolución de los pacientes con signos de encefalopatía hiperbilirrubinemica durante la hospitalización, comparando con los datos de la literatura. 


\section{Pacientes y Método}

Estudio descriptivo, retrospectivo. Se incluyeron a los recién nacidos hospitalizados en el servicio de Neonatología del Complejo Asistencial Dr. Sótero del Río (CASR), entre el 01 de enero del 2013 y el 31 de diciembre del 2016, con diagnóstico al alta de hiperbilirrubinemia, cuyo valor máximo de bilirrubina sérica total fue mayor o igual a $20 \mathrm{mg} / \mathrm{dl}$,a partir de la base electrónica de egresos. Con esta información se revisaron las epicrisis de los recién nacidos. Se excluyeron a los RN en los que no fue posible obtener acceso a epicrisis electrónica.

Durante el periodo de estudio los niveles de bilirrubina se analizaron mediante el mismo método, Bilirrubina total: punto final ascendente/Ión diazonio.

Se estratificó a los RN por valores de bilirrubina en dos niveles, mayor o igual a de $20 \mathrm{mg} / \mathrm{dl}$ y mayor o igual a $25 \mathrm{mg} / \mathrm{dl}$; por edad de ingreso, como menor o igual a tres días, entre 4 y 7 días y mayor o igual a 8 días y además, por edad gestacional. Se evaluaron factores de riesgo para hiperbilirrubinemia severa: sexo, edad gestacional, adecuación del peso al nacer, baja de peso excesiva, incompatibilidad de grupo y $\mathrm{Rh}$, presencia de cefalohematomas, poliglobulia, sepsis, asfixia perinatal, infección urinaria e hipotiroidismo, diagnósticos definidos en la tabla 1.

Se registró la presencia de síntomas neurológicos durante la hospitalización, compatibles con encefa- lopatía bilirrubínica aguda y se revisaron los datos de atención ambulatoria en neurología infantil en el CASR de estos pacientes.

Se compararon los datos con test exacto de Fisher, $\chi^{2}$ y riesgo relativo (RR) en una base de excel, con un error alfa de un $\mathrm{p}<0,05$.

El estudio fue revisado y aprobado por el Comité de Ética e Investigación del Servicio de Salud Metropolitano Sur-Oriente.

\section{Resultados}

En el periodo estudiado nacieron 25.288 recién nacidos vivos (RNV), $48,3 \%$ de sexo femenino y $51,6 \%$ de sexo masculino. Se hospitalizaron $599 \mathrm{RN}$ con bilirrubina mayor o igual a $20 \mathrm{mg} / \mathrm{dl}$, de los que se excluyeron 6 casos, dado que no hubo acceso a la epicrisis electrónica. Con los 593 casos, se observó una incidencia de $2,3 \%$ o 1 caso por cada 42 RNV. 59 RN presentaron niveles de bilirrubina mayor o igual a $25 \mathrm{mg} / \mathrm{dl}$, con una incidencia de $0,23 \%$ o 1 caso por $428 \mathrm{RNV}$. Se rehospitalizaron por HBR severa $53 \mathrm{RN}$ ( $9 \%$ de los casos), 6 de los cuales presentaron, en ambas hospitalizaciones, niveles de bilirrubina mayor a $20 \mathrm{mg} / \mathrm{dl}$.

Del total de pacientes, el $80,6 \%$ fueron de término, $18,7 \%$ correspondieron a prematuros tardíos y $0,7 \%$ a moderados. No hubo casos de hiperbilirrubinemia severa en los menores de 32 semanas de edad gestacional.

En la figura 1 se describe el riesgo de bilirrubinemia

Tabla 1. Definiciones operacionales

\begin{tabular}{|c|c|}
\hline Término & Definición \\
\hline RN prematuro extremo & Nacido antes de 32 semanas de gestación \\
\hline RN prematuro moderado & Nacido entre 32 y 33 semanas de gestación \\
\hline RN prematuro tardío & Nacido entre 34 y 36 semanas de gestación \\
\hline RN término & Nacido entre 37 y 41 semanas de gestación \\
\hline Pequeño para edad gestacional ${ }^{19}$ & Peso nacimiento menor a percentil 10 para edad gestacional \\
\hline Adecuado para edad gestacional ${ }^{19}$ & Peso nacimiento entre percentil 10 a 90 para edad gestacional \\
\hline Grande para edad gestacional ${ }^{19}$ & Peso nacimiento mayor a percentil 90 para edad gestacional \\
\hline Baja de peso excesiva & $\begin{array}{l}\text { Baja de peso entre } 8 \text { a } 12 \% \text { del peso de nacimiento o no recuperación del peso de nacimiento a los } \\
7 \text { días de vida }\end{array}$ \\
\hline Baja severa de peso & Baja de peso mayor a $12 \%$ del peso de nacimiento \\
\hline Incompatibilidad de grupo clásico & $\begin{array}{l}\text { Grupos sanguíneos entre recién nacido y madre sugerentes, con Test de Coombs directo positivo o } \\
\text { recuento de reticulocitos mayor a } 6 \% \text { en el hemograma del RN }\end{array}$ \\
\hline Incompatibilidad por Rh & Madre Rh negativa sensibilizada y su RN Rh positivo con Coombs directo positivo \\
\hline Poliglobulia & Hematocrito igual o mayor a $65 \%$ \\
\hline Sepsis & $\begin{array}{l}\text { Hemocultivos positivos o tratamiento antibiótico por } 5 \text { o más días, con clínica y laboratorio sugerente } \\
\text { a pesar de hemocultivos negativos }\end{array}$ \\
\hline Infección urinaria & $\begin{array}{l}\text { Urocultivo positivo por sondeo a micro organismo patogénico mayor a } 10.000 \text { unidades formadoras } \\
\text { de colonias }\end{array}$ \\
\hline
\end{tabular}

RN: recien nacido. 
entre 20 y $24,9 \mathrm{mg} / \mathrm{dl}$ y mayor de $25 \mathrm{mg} / \mathrm{dl}$ según edad gestacional al nacer. El mayor riesgo de bilirrubina entre 20 y $25 \mathrm{mg} / \mathrm{dl}$ se observó a las 35 semanas (uno de cada $16 \mathrm{RN}$ de esa edad gestacional) y mayor de $25 \mathrm{mg} /$ $\mathrm{dl}$, a las 36 semanas (uno de cada $85 \mathrm{RN}$ ). Comparando con el RN de término, los pretérmino tuvieron más riesgo de HBR severa, con un RR 1,78 (IC 95\% 1,452,17), especialmente los pretérminos tardíos, entre 34 y 36 semanas, con un RR de 2,39 (IC 95\% 1,96-2,93).

En la tabla 2 se muestra las características del grupo según edad de hospitalización y causas asociadas. El 19,4\% de los casos fueron menores 37 semanas. El sexo masculino aumenta con los días de presentación, globalmente el sexo masculino presenta más riesgo que el sexo femenino, con un RR de 1,22 (IC 95\% 1,041,44). Los casos de hiperbilirrubinemia mayor de 25 $\mathrm{mg} / \mathrm{dl}$ se observaron mayoritariamente en menores de 8 días de vida.

En los RN que se hospitalizaron antes de los 4 días de vida, el principal factor de riesgo fue la incompatibilidad de grupo clásico, con un RR de 3,78 (IC 95\% 1,84$7,78)$. En los RN que se hospitalizan desde los 4 días de vida, el factor de riesgo de mayor importancia fue la baja de peso excesiva, con un RR 2,61 (IC 95\% 1,74-3,92) en
Figura 1. Riesgo de bilirubinemia (\%) de 20 a 25 y mayor de $25 \mathrm{mg} / \mathrm{dl}$ según semanas de edad gestacional al nacer.

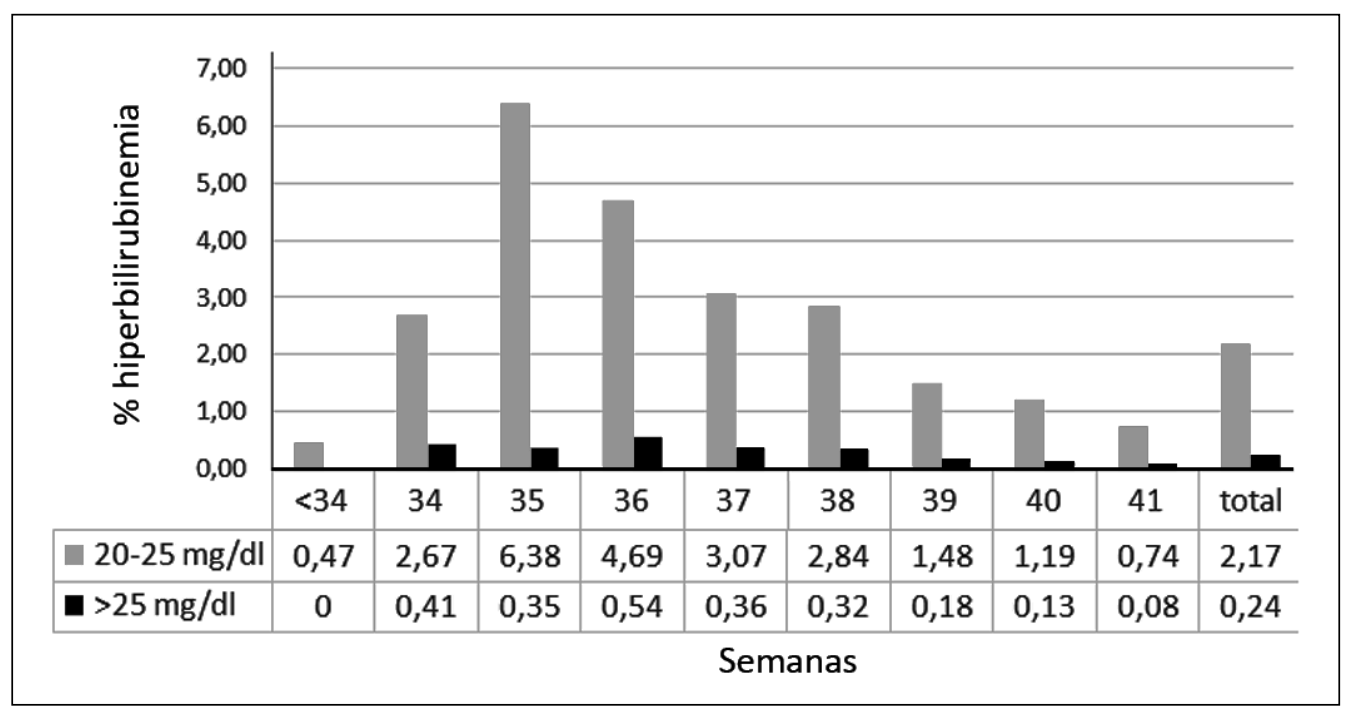

Tabla 2. Características principales de RN hospitalizados por HBR severa, según edad de ingreso

\begin{tabular}{lccc}
\hline Característica & $\leq 3$ días & $4-7$ días & $\geq 8$ días \\
\hline Total RN & 186 & 288 & 119 \\
Sexo masculino (\%) & $51,1 \%$ & $59,0 \%$ & $59,7 \%$ \\
Edad gestacional promedio sem (ds) & $37,9(1,6)$ & $37,7(1,6)$ & $37,8(1,6)$ \\
Prematuros n (\%) & $37(19,9 \%)$ & $58(20,1 \%)$ & $20(16,8 \%)$ \\
Edad al ingreso promedio días (ds) & $2,5(0,65)$ & $5,31(1,1)$ & $9,89(2,8)$ \\
Pequeño para la edad gestacional & $14(7,5 \%)$ & $16(5,6 \%)$ & $9(7,6 \%)$ \\
Grande para la edad gestacional & $23(12,4 \%)$ & $21(7,3 \%)$ & $10(8,4 \%)$ \\
Baja de peso 8-12\% (\%) & $34,4 \%$ & $24 \%$ & $12,6 \%$ \\
Baja de peso > 12\% (\%) & $3,2 \%$ & $10,8 \%$ & $5(4,2 \%)$ \\
Incompatibilidad de Grupo Clásico & $19(10,2 \%)$ & $6(2,1 \%)$ & $3(2,5 \%)$ \\
Incompatibilidad por Rh & $3(1,6 \%)$ & $3(1 \%)$ & $8 / 27$ \\
Infección urinaria n/total ex & $1 / 4$ & $4 / 17$ & $1 / 22$ \\
Hipotiroidismo n/total ex & $0 / 3$ & $0 / 7$ & 1 \\
Exsanguineotransfusión N(\%) & $6(3,2 \%)$ & $5(1,6 \%)$ & $(2)$ \\
Inmunoglobulina EV & 13 & $(1)$ & \\
\hline
\end{tabular}

RN: recién nacido; HBR: hiperbilirubinemia; ex: exámenes; ds: días. 
los RN entre 4 y 7 días y un RR 6,71 (IC 95\% 4,59-9,81) en los con edad mayor o igual a 8 días de vida.

La infección urinaria fue buscada en 44 casos y un $27 \%$ tuvo urocultivo positivo. Destaca por su importancia un caso de hipotiroidismo. En 75 RN (12,6\%) se asoció más de un factor de riesgo, encontrando con mayor frecuencia la conjunción de prematurez con baja de peso excesiva.

Otros factores de riesgo, como la adecuación de peso al nacer, la presencia de cefalohematomas, poliglobulia, sepsis o asfixia, no se relacionaron con mayor riesgo de HBR severa.

Recibieron fototerapia todos los $\mathrm{RN}$ hospitalizados por HBR severa, $16 \mathrm{RN}$ recibieron inmunoglobulina y
12 exsanguineotransfusión. De los $\mathrm{RN}$ que recibieron inmunoglobulina, 3 casos correspondieron a re hospitalizaciones, recibiendo este fármaco durante la primera hospitalización.

En la tabla 3 se describe el subgrupo con HBR mayor de $25 \mathrm{mg} / \mathrm{dl}$ en los mismos períodos de ingreso, con mayor predominio de sexo masculino y de incompatibilidad.

Los RN con bilirrubina igual o mayor a $25 \mathrm{mg} / \mathrm{dl}$ presentaron un examen neurológico anormal en $10 \mathrm{de}$ 59 casos. Se describió hipotonía leve en 2 casos, hipertonía leve en 6 y encefalopatía en 2 casos, lo que determina una incidencia de encefalopatía bilirrubinica aguda de 0,04\% del total de RN. En la tabla 4 se des-

Tabla 3. Características principales del subgrupo de RN hospitalizados por HBR $\geq \mathbf{2 5} \mathbf{m g} / \mathrm{dl}$, según edad de ingreso

\begin{tabular}{lccc}
\hline Característica & $\leq 3$ días & $4-7$ días & $\geq 8$ días \\
\hline Total RN & 11 & 32 & 16 \\
Sexo masculino (\%) & $63,6 \%$ & $56,3 \%$ & $62,5 \%$ \\
Edad gestacional promedio sem (ds) & $38,09(1,7)$ & $37,91(1,4)$ & $37,68(1,6)$ \\
Prematuros n (\%) & $2(18,2 \%)$ & $4(12,5 \%)$ & $3(18,8 \%)$ \\
Edad al ingreso promedio días (ds) & $2,4(0,7)$ & $5,6(1,2)$ & $9,2(0,9)$ \\
Pequeño para la edad gestacional & 0 & $1(3,1 \%)$ & 0 \\
Grande para la edad gestacional & $2(18,2 \%)$ & $2(6,3 \%)$ & $2(12,5 \%)$ \\
Baja de peso 8-12\% & $45,5 \%$ & $21,9 \%$ & $50 \%$ \\
Baja de peso > 12\% & $0 \%$ & $18,8 \%$ & $12,5 \%$ \\
Incompatibilidad de Grupo Clásico & $3(27,3 \%)$ & $4(12,5 \%)$ & $2(12,5 \%)$ \\
Incompatibilidad por Rh & 0 & $4(12,5 \%)$ & $1(6,3 \%)$ \\
Infección urinaria n/total ex & $0 / 1$ & $3 / 9$ & $0 / 3$ \\
Hipotiroidismo n/total ex & $0 / 1$ & $0 / 6$ & $0 / 3$ \\
Exsanguineotransfusión n (\%) & $3(27,3 \%)$ & $5(15,6 \%)$ & $1(6,3 \%)$ \\
Inmunoglobulina EV & $2 / 11$ & $0 / 16$ & $0 / 16$ \\
\hline
\end{tabular}

RN: recién nacido; HBR: hiperbilirubinemia; ex: exámenes; ds: días.

Tabla 4. Casos en que se describió clínica sugerente de encefalopatía bilirrubinica aguda

\begin{tabular}{|c|c|c|c|c|c|c|c|c|c|}
\hline PN & $\mathrm{EG}$ & Edad & $\% \mathrm{PN}$ & Inc & Uro & Bili & Ext & Signos neurologicos & Evolución neurológica \\
\hline 3.155 & 37 & 7 & 10,0 & & $(-)$ & 30 & & Hipotonía & ¿? \\
\hline 3.400 & 38 & 5 & 2,9 & & $(+)$ & 27,6 & & Hipotonía & Normal \\
\hline 2.310 & 35 & 9 & 3,0 & & & 28,5 & & Hipertonía & Normal \\
\hline 2.545 & 37 & 4 & 5,5 & & $(+)$ & 29 & + & Hipertonía & $\begin{array}{l}\text { Sind. distónico } \\
\text { Bera N }\end{array}$ \\
\hline 3.720 & 38 & 6 & 6,5 & $0-A$ & & 30,8 & + & Hipertonía & $\begin{array}{l}\text { Retraso lenguaje } \\
\text { Bera N Motor N }\end{array}$ \\
\hline 3.530 & 39 & 9 & 15,2 & & $(-)$ & 28,9 & & Hipertonía & ¿? \\
\hline 3.615 & 40 & 1 & 11,3 & $0-B$ & & 25,4 & + & Hipertonía & Normal \\
\hline 3.580 & 38 & 6 & 14,9 & $0-B$ & & 32,4 & + & Hipertonía & Normal Bera N \\
\hline 3.390 & 40 & 6 & 8,6 & & & 26,6 & & Hipotonía llanto agudo opistotono & Retraso psicomotor \\
\hline 3.180 & 38 & 6 & 11,0 & $\mathrm{Rh}$ & & 29,1 & + & Hipotonía succión débil & Normal \\
\hline
\end{tabular}

PN: peso de nacimiento; EG: edad gestacional; Edad: en días; \%PN :porcentaje del PN al ingreso; Inc: incompatibilidad Sanguínea; Uru: urocultivo; Bili: valor máximo de bilirrubina mg/dl; ExT: exsanguineotransfusión; Bera: potenciales evocados Auditivos; N: normal; ¿? Sin información. 
criben los casos y sus factores asociados. Dos pacientes tienen Resonancia nuclear magnética, informadas como normales. En 5 casos se realizó exsanguineotransfusión, en los otros la bilirrubina disminuyó significativamente con las primeras horas de fototerapia e hidratación. No hay seguimiento en 2 casos y en 3 casos hay un cuadro clínico compatible con encefalopatía crónica por bilirrubina, con una incidencia de 11,8 /100.000 recién nacidos.

\section{Discusión}

La hiperbilirrubinemia severa, definida como niveles de bilirrubina total mayor $25 \mathrm{mg} / \mathrm{dl}$ en un recién nacido de término o mayor de $20 \mathrm{mg} / \mathrm{dl}$ en un prematuro mayor o igual a 34 semanas, representa un factor de riesgo para la aparición de neurotoxicidad y secuelas secundarias a la impregnación de tejido nervioso por bilirrubina, en las que destacan trastornos visuales, auditivos, y signos de parálisis cerebral extrapirami$\mathrm{dal}^{4,6,8,9,21,22}$. Es una preocupación permanente en Neonatología.

La incidencia de HBR severa fue sorprendentemente alta, desde casi el doble a 40 veces a lo descrito en la literatura en países desarrollados ${ }^{5,10,21}$. En el análisis global se observó 1 caso/47 RN con HBR entre 20-25 mg/dl; y 1/428 RN con HBR mayor 25 mg/dl. Datos de California muestran bilirrubinemia mayor de $20 \mathrm{mg} / \mathrm{dl}$ en $1 / 72$ y mayor de $25 \mathrm{mg} / \mathrm{dl}$ en $1 / 1430 \mathrm{RN}$ vivos $^{29}$. En países desarrollados se observa entre $1 / 2.000$ - 1/10.000 casos de RN con HBR mayor de $25 \mathrm{mg} / \mathrm{dl}$, incluso se ha reportado 1/18.000 $\mathrm{RN}$ en Israel, que cuenta con una coordinación fluida con la atención primaria y un protocolo detallado de prevención y detección ${ }^{28}$. En países de ingresos bajos, como África y Asia, la incidencia reportada de $\mathrm{HBR}$ mayor de $25 \mathrm{mg} / \mathrm{dl}$ varía entre 4 y $46 \%{ }^{23}$. No encontramos información sobre su incidencia en Latinoamérica.

Los principales factores de riesgo para desarrollar HBR severa fueron el sexo masculino, prematurez, baja de peso excesiva e incompatibilidad de grupo clásico. El grupo de mayor riesgo lo constituyen los prematuros tardíos, entre 34-36 semanas de edad gestacional, que tienen un RR de 2,39 (IC 95\% 1,96-2,93), respecto a los de término, similar a lo descrito en la literatura $9,10,15-17,23,24$.

El máximo ascenso de la bilirrubina en el RN de término ocurre entre los 3 a 5 días, cuando el RN ya ha sido dado de alta, por lo que el seguimiento en niños con riesgo es importante. Además, la máxima pérdida de peso normal se produce a los 3 días, con una baja promedio entre el 6 a $8 \%$ del peso de nacimiento; en los recién nacidos que han perdido más del $10 \%$ del peso es necesaria la evaluación, para eventualmente suplementar la lactancia y evaluar la presencia de $\mathrm{HBR}^{3,9}$.
La relación entre HBR e infección urinaria aún no se ha comprendido por completo. Se ha descrito que alrededor del 8\% de HBR en las que no se logra encontrar causa, presentan infección urinaria, especialmente por Escherichia coli ${ }^{25}$. Dado el carácter retrospectivo del trabajo no se obtuvo urocultivo en todos los pacientes, por lo que no es posible determinar la incidencia de infección urinaria en este grupo. El urocultivo por sondeo fue positivo en el $27 \%$ de los casos evaluados y constituye una morbilidad importante de descartar en la hiperbilirrubinemia tardía que tenga síntomas sugerentes: fiebre, baja de peso, alteración de procalcitonina.

La evaluación de hipotiroidismo debe reservarse para el caso sin elementos que expliquen la hiperbilirrubinemia y especialmente si hay elementos de un defecto primario, que no es detectable con el tamizaje.

Doce casos requirieron exsanguineotransfusión, 47/100.000 RNV, comparado con 1,9 a 3,6/100.000 RNV en California ${ }^{22}$. De los 10 casos sintomáticos sólo 5 fueron tratados con exsanguineotransfusión, ya que mientras se preparaba ésta se observó descenso significativo de la bilirrubina, o la alteración neurológica fue posterior, pero frente a un caso sintomático la terapia recomendada es la exsanguineotransfusión ${ }^{15}$.

Si bien la encefalopatía aguda neonatal por bilirrubina ha disminuido dramáticamente por el adecuado control y tratamiento de la madre Rh negativo para prevenir su sensibilización, se observa cierto aumento del riesgo con el aumento de las altas precoces, la falta de advertencia a los padres sobre el riesgo de la hiperbilirrubinemia y a la falta de control oportuno a nivel ambulatorio, especialmente de los pacientes de riesgo, como son los prematuros tardíos y los RN de término precoces ${ }^{7,9,26}$. Además las alteraciones neurológicas y auditivas pueden ser transitorias o de aparición tardías, por lo que es muy importante el seguimiento a corto y largo plazo de los RN expuestos a altos niveles de bilirrubina ${ }^{7}$.

La incidencia de encefalopatía aguda y crónica ha disminuido de forma importante. Se ha estimado una incidencia de 0,3 a 1 en 10.000 RNV para la encefalopatía aguda y alrededor de 1 en 100.000 para la crónica $^{4,9,13,14}$. En esta revisión se observaron 4 casos en 10.000 de encefalopatía aguda y 11,8 casos en 100.000, de crónica. No hay datos nacionales de encefalopatía crónica por bilirrubina, pero una publicación nacional muestra que no ha desaparecido ${ }^{30}$.

Las limitaciones de este estudio son que es retrospectivo y los datos se obtuvieron de las epicrisis, donde se podría haber omitido información, como la búsqueda de otros diagnósticos si la causa de la hiperbilirrubinemia fue poco clara. La fecha de ingreso no fue necesariamente a causa de hiperbilirrubinemia y el descenso de peso corresponde al mínimo peso observado. 
Datos interesantes como el tipo de alimentación previo a la hospitalización y la percepción de la madre respecto a la ictericia, no estaban disponibles. Todos los casos de hiperbilirrubinemia mayor de $25 \mathrm{mg} / \mathrm{dl}$ son derivados a neurología infantil y a un policlínico de seguimiento de encefalopatía, pero la inasistencia a corto plazo y la falta de un sistema de rescate ha impedido contar con información completa de los pacientes. De los 10 pacientes identificados con signos de encefalopatía aguda, se obtuvo información sobre seguimiento en 8 , de éstos, hay un caso identificado con encefalopatía crónica y 2 adicionales probables.

La fortaleza de este estudio es mostrar que el riesgo de daño neurológico por bilirrubina continúa ocurriendo, por lo que es importante la detección y tratamiento oportuno de la HBR. La hiperbilirrubinemia severa es un evento centinela que hay que evitar, por lo que, hay que establecer normativas a seguir según edad gestacional, horas de vida, y el seguimiento pertinente, diferentes que los previamente utilizados, ampliando los rangos de control. El prematuro tardío debe tener control de bilirrubinemia al alta, independientemente de si tiene ictericia, y seguimiento.

La determinación de la bilirrubina transcutánea en forma seriada usando nomogramas conocidos permitiría alertar, sobretodo cuando hay cambios importantes de los niveles ${ }^{31}$. Para el riesgo tardío es necesaria una mayor coordinación con nivel primario y el control precoz postalta, especialmente de los prematuros tardíos, cuyo control debiera ser antes de las $72 \mathrm{~h}$ del egreso, como se ha señalado en el nuevo programa de atención infantil del Ministerio de Salud ${ }^{32}$. Con la instalación de un sistema de vigilancia y de intervención, se podría disminuir el riesgo de hiperbilirrubinemia severa y sus potenciales secuelas ${ }^{28,29}$.
En conclusión, la incidencia de HBR severa en este periodo fue inaceptablemente alta. Los principales factores de riesgo para desarrollar HBR severa fueron el sexo masculino, prematurez, baja de peso excesiva e incompatibilidad de grupo clásico. Se deben modificar prácticas clínicas de control de bilirrubinemia, especialmente para los prematuros tardíos, de criterio de alta de fototerapia y establecer un protocolo prospectivo de vigilancia de HBR severa, que por sus consecuencias, debiera ser considerada como un evento centinela vigilado a nivel nacional.

\section{Responsabilidades Éticas}

Protección de personas y animales: Los autores declaran que los procedimientos seguidos se conformaron a las normas éticas del comité de experimentación humana responsable y de acuerdo con la Asociación Médica Mundial y la Declaración de Helsinki.

Confidencialidad de los datos: Los autores declaran que han seguido los protocolos de su centro de trabajo sobre la publicación de datos de pacientes.

\section{Derecho a la Privacidad y Consentimiento Informa- do: Los autores declaran que la información ha sido obtenida de datos previos en forma anonimizada, por lo cual el Comité de Ética de Investigación en uso de sus facultades, ha eximido de la obtención de un con- sentimiento informado, lo cual consta en el acta res- pectiva.}

\section{Conflicto de intereses}

Los autores declaran no tener conflicto de intereses.

\section{Referencias}

1. American Academy of Pediatrics, Provisional Committee for Quality Improvement. Practice parameter: management of hyperbilirubinemia in the healthy term newborn. Pediatrics. 1994;94:558-65.

2. Chou SC, Palmer RH, Ezhuthachan S, et al. Management of hyperbilirubinemia in newborns: measuring performance by using a benchmarking model. Pediatrics. 2003;112:1264-73.

3. Yang WC, Zhao LL, Li YC, et al. Bodyweight loss predicting neonatal hyperbilirubinemia 72 hours after birth in term newborn infants.BMC Pediatr. 2013;13:145.

4. Maisels MJ, Watchki JF, Bhutani VK, Stevenson DK. An approach to the management of hyperbilirubinemia in the preterm infants less than 35 weeks of gestation. J Perinatol. 2012; 32(9):6604.

5. Bhutani VK, Johnson-Hamerman L. The clinical syndrome of bilirubin induced neurologic dysfunction. Semin Fetal Neonatal Med. 2015;20(1):6-13.

6. Ullah S, Rahman K, Hedayati M. Hyperbilirubinemia in neonates: Types, Causes, Clinical Examinations, Preventive Measures and Treatments: A Narrative Review Article. Iran J PublicHealth. 2016;45(5):558-68.

7. Olds C, Oghalai JS. Bilirubin-Induced Audiologic Injury in Preterm Infants. Clin Perinatol. 2016;43(2):313-23.

8. Olusanya BO, Iskander IF, Slusher TM, Wennberg RP. A decision making tool for Exchange transusions in infant with severe hyperbilirubinemia in resource limited settings. JPerinatol. 2016;36(5):338-41.

9. Canadian Paediatric Society, Fetus and Newborn Committee. Guidelines for detection, management and prevention of hyperbilirubinemia in term and late preterm newborn infants ( 35 or more weeks' gestation). Paediatr Child Health. 2007;12:1B-12B. Available at: www.cps. ca/english/ statements.

10. Bhutani VK, Johnson LH, Maisels MJ, et al. Kernicterus: epidemiological stategies for its prevention through systems based approaches. J Perinatol. 2004;24(10):65062.

11. Kaplan M, Bromiker R, Hammerman C. Severe neonatal hyperbilirubinemua and kernicterus: are still problems in the third millennium?.Neonatology. 2011;100(4):354-62. 
12. Brites D, Fernandes A. Bilirubin induced neural impairment: a special focus of myelination, age related window of susceptibility and associated comorbidities. Semin Fetal Neonatal Med. 2015;20(1):14-9.

13. Centers for Disease Control and Prevention (CDC). Kernicterus in full term infants-United States, 1994-1998. MMWR Morb Mortal Wkly Rep. 2001;50:491-4.

14. Maisels MJ, Newman TB. Jaundice in full-term and near-term babies who leave the hospital within 36 hours. The pediatrician's nemesis. Clin Perinatol 1998;25:295-302.

15. American Acdemy of Pediatrics Subcommittee of Hyperbilirubinemia. Management of hyperbilirubinemia in infant 35 or more weeks of gestation. Pediatrics. 2004;114(1):297-316.

16. Keren R, Luan X, Friedman S, Saddlemire S, Cnaan A, Bhutani VK. A comparison of alternative riskassessment strategies for predicting significant neonatal hyperbilirubinemia in term and near-term infants. Pediatrics.2008;121(1):e170-9.

17. Stevenson DK, Vreman HJ, Wong RJ. Bilirrubin production and the risk of bilirubin neurotoxicity. Semin Perinatol. 2011;35(3):121-6.

18. Maisels MJ, Bhutani VK, Bogen D,
Newman TB, Stark AR, Watchko JF. Hyperbilirubinemia in the newborn infant $>$ or $=35$ weeks' gestation: an update with clarifications. Pediatrics. 2009;124(4):1193-8.

19. Milad M, Novoa JM, Fabres J, Samamé MM, Aspillaga C. Recomendación sobre Curvas de Crecimiento Intrauterino. Rev Chil Pediatr. 2010;81(3):264-74.

20. Hankins GD, Speer M. Defining the pathogenesis and pathophysiology of neonatal encephalopathy and cerebral palsy. Obstet Gynecol. 2003;102(3):62836.

21. Bhutani VK, Johnson L. Kernictus in the 21st century: frequently ansked questions. J Perinatol. 2009;29 Suppl1:S20-4.

22. Bhutani VK, Meng NF, Knauer Y, Danielsen BH, Wong RJ, Stevenson DK, Gould JB. Extreme hyperbilirubinemia and rescue Exchange transfusion in California from 2007 to 2012. J Perinatol. 2016;36(10):853-7.

23. Olusanya BO, Osibanjo FB, Slusher TM. Risk factors for severe neonatal hyperbilirubinemia in low and middle-income countries: a systematic review and meta analysis. PloSOne. 2015;12:10(2):e0117229.

24. Maisels MJ. Neonatal hyperbilirubinemia and kernicterus-not gone but sometimes forgotten.Early Hum Dev. 2009;85(11):727-32.
25. Mutlu M, Cayir Y, Asian Y. Urinary tract infections in neonates with jaundice in their first two weeks of life. World J Pediatr. 2014;10(2):164-7.

26. Wallenstein MB, BhutaniVK. Jaundice and kernicterus in the moderately preterm infant. Clin Perinatol. 2013;40(4):679-88.

27. Olds C, Oghalai JS. Audiologic impairment associated with bilirubininduced neurologic damage. Semin Fetal Neonatal Med. 2015; 20(1):42-6.

28. Kaplan M, Bromiker R, Schimmel MS, Algur N, Hammerman C. Evaluation of discharge management in the prediction of hyperbilirubinemia: the Jerusalem experience. J Pediatr. 2007;150(4):412-7.

29. Wickremasinghe AC, Kuzniewicz MW, Newman TB. Black race is not protective against hazardous bilirubin levels. J Pediatr 2013;162(5):1068-89.

30. Hernández M, Schmidt MI, Huete I. Encefalopatía por Kernicterus. Serie clínica. Rev Chil Pediatr 2013;84(6):65966.

31. Bromiker R, Goldberg A, Kaplan M. Israel transcutaneous bilirubin normogram predicts significantHyperbilirubinemia. Journal of Perinatology 2017;00:1-4.

32. Minsal Chile. Programa Nacional de Salud de la Infancia con Enfoque Integral. Ed Valente. ISBN 978-956-348-03372013. 